\title{
Arithmetic progressions in sumsets
}

\author{
by \\ IMre Z. RuZSA* (Budapest)
}

1. Introduction. Let $A, B \subset[1, N]$ be sets of integers, $|A|=|B|=c N$. Bourgain [2] proved that $A+B$ always contains an arithmetic progression of length $\exp (\log N)^{1 / 3-\varepsilon}$. Our aim is to show that this is not very far from the best possible.

Theorem 1. Let $\varepsilon$ be a positive number. For every prime $p>p_{0}(\varepsilon)$ there is a symmetric set $A$ of residues $\bmod p$ such that $|A|>(1 / 2-\varepsilon) p$ and $A+A$ contains no arithmetic progression of length

$$
\exp (\log p)^{2 / 3+\varepsilon} \text {. }
$$

A set of residues can be used to get a set of integers in an obvious way. Observe that the $1 / 2$ in the theorem is optimal: if $|A|>p / 2$, then $A+A$ contains every residue.

Acknowledgement. I profited much from discussions with E. Szemerédi; he directed my attention to this problem and to Bourgain's paper.

2. The construction. In this section we describe the set $A$ of Theorem 1 and prove its properties, assuming Theorems 2 and 3 (to be stated below) which will be proved in Sections 3 and 4 .

Our construction goes as follows. Take $k$ residues $a_{1}, \ldots, a_{k} \in \mathbf{Z}_{p}$ and write

$$
F(x)=\sum e\left(a_{j} x / p\right), \quad f(x)=\operatorname{Re} F(x)=\sum \cos \left(2 \pi a_{j} x / p\right) ;
$$

here, as usual, $e(t)=\exp 2 \pi i t$. Take a $Q>0$ and set

$$
A=\{x: f(x)>Q\} .
$$

* This paper was presented at the CBMS regional conference in Manhattan, Kansas, May 1990. Participation was supported by Hungarian National Foundation for Scientific Research, Grant No. 1811 and NSF. 
$A$ is a symmetric set of residues. If $x, y \in A$, then we have

$$
\begin{aligned}
2 Q & <\operatorname{Re} \sum\left(e\left(a_{j} x / p\right)+e\left(a_{j} y / p\right)\right) \\
& =\operatorname{Re} \sum e\left(a_{j} y / p\right)\left(1+e\left(\frac{a_{j}(x-y)}{p}\right)\right) \\
& \leq \sum\left|1+e\left(\frac{a_{j}(x-y)}{p}\right)\right| .
\end{aligned}
$$

Consequently, $A-A$ (which is equal to $A+A$ by the symmetry) will be disjoint from the set

$$
H=\left\{h: \sum\left|1+e\left(a_{j} h / p\right)\right|<2 Q\right\} .
$$

Our task is to find $a_{1}, \ldots, a_{k}$ and $Q$ so that $|A|>(1 / 2-\varepsilon) p$ and $H$ intersects every not too short arithmetic progression.

For a typical choice of $a_{1}, \ldots, a_{k}$, the functions $e\left(a_{j} x / p\right)$ will be almost independent, thus $f(x)$ has approximately a normal distribution with variance $k / 2$; hence $|A| \sim p / 2$ will hold if $Q=o(\sqrt{k})$. We formulate this exactly as follows.

2.1. Definition. We call the sequence $a_{1}, \ldots, a_{k} \in \mathbf{Z}_{p} K$-independent for a number $K>0$ if the equation

$$
\sum a_{j} x_{j} \equiv 0(\bmod p)
$$

has no solution with $0<\sum\left|x_{j}\right| \leq K$.

TheOREM 2. Let $a_{1}, \ldots, a_{k}$ be a $K$-independent sequence of residues $\bmod p, c_{1}, \ldots, c_{k}$ real numbers, $\sum c_{j}^{2}=2 \sigma^{2}>0, \max \left|c_{j}\right|=\Delta, \sum\left|c_{j}\right|=S$. Put

$$
f(x)=\sum c_{j} \cos \left(2 \pi a_{j} x / p\right) .
$$

We have uniformly in $t$

$$
\frac{1}{p} \sum_{f(x) \leq t \sigma} 1-\Phi(t) \ll\left(\frac{\Delta}{\sigma}\right)^{2}+\min \left(\frac{1}{\sqrt{K}}, \frac{S}{\sigma K}\right),
$$

where $\Phi$ is the standard normal distribution. In particular, if $c_{j}=1$ for all $j$, then

$$
\frac{1}{p} \sum_{f(x) \leq Q} 1-\Phi\left(\sqrt{\frac{2}{k}} Q\right) \ll \frac{1}{k}+\min \left(\frac{1}{\sqrt{K}}, \frac{\sqrt{k}}{K}\right) .
$$

Theorem 2 will be proved in Section 4 .

The set $H$ is defined in terms of the function $g(h)=\sum\left|1+e\left(a_{j} h / p\right)\right|$ which is more difficult to handle because of the || sign. We may try a 
square-mean inequality:

$$
g(h) \leq \sqrt{k \sum\left|1+e\left(a_{j} h / p\right)\right|^{2}}=\sqrt{2 k(k+f(h))} .
$$

So, to guarantee a small value of $g(h)$ it is sufficient to have $f(h) \approx-k$. To ensure this we need a stronger assumption than $K$-independence.

2.2. Definition. We call the sequence $a_{1}, \ldots, a_{k} \in \mathbf{Z}_{p} K, L$-separated for $K, L>0$ if the equation

$$
y+\sum a_{j} x_{j} \equiv 0(\bmod p)
$$

has no solution with $0<\sum\left|x_{j}\right| \leq K,|y| \leq L$.

TheOREM 3. Let $a_{1}, \ldots, a_{k}$ be a K,L-separated sequence of residues $\bmod p, c_{1}, \ldots, c_{k}$ real numbers, $\sum\left|c_{j}\right|=S$. Put

$$
f(x)=\sum c_{j} \cos \left(2 \pi a_{j} x / p\right) .
$$

Suppose $k \geq 4,0<\delta<1 / 2$. If

$$
K \geq \frac{4 k}{\delta} \log \frac{2}{\delta}
$$

and

$$
T \geq \frac{4 p}{L}(2 / \delta)^{2 k},
$$

then among any $T$ consecutive values of $x$ there is always one for which $f(x)>S(1-\delta)$ as well as one with $f(x)<-S(1-\delta)$.

This theorem will be proved in Section 3.

2.3. Corollary. Let $a_{1}, \ldots, a_{k}$ be a $K, L$-separated sequence of residues $\bmod p, g(h)=\sum\left|1+e\left(a_{j} h / p\right)\right|, K>4 k$. If (2.9) and (2.10) are satisfied, then among any $T$ consecutive values of $x$ there is always one for which $g(h)<k \sqrt{2 \delta}$.

Proof. This follows immediately from the previous theorem and inequality (2.7).

This result is not directly applicable to our problem, since we need to find small values of $g(h)$ in every arithmetic progression, not just in those with difference 1 . A sequence such that $a_{1} d, \ldots, a_{k} d$ is $K, L$-separated for every $d \neq 0$ would suffice, but such a sequence does not exist. Fortunately, a somewhat weaker assumption also works.

2.4. Definition. We call the sequence $a_{1}, \ldots, a_{k} \in \mathbf{Z}_{p} K, L, m$-quasiseparated if $m$ of them can be omitted so that the remaining $k-m$ are $K, L$-separated. 
2.5. Statement. Let $a_{1}, \ldots, a_{k}$ be a $K, L, m$-quasiseparated sequence of residues $\bmod p, g(h)=\sum\left|1+e\left(a_{j} h / p\right)\right|, K>4 k$. If (2.9) and (2.10) are satisfied, then among any $T$ consecutive values of $x$ there is always one for which $g(h)<2 m+k \sqrt{2 \delta}$.

Proof. Put $g=g_{1}+g_{2}$, where $g_{1}$ contains the $m$ omitted terms, and $g_{2}$ the remaining $k^{\prime}=k-m$. We apply Corollary 2.3 to $g_{2}$. If (2.9) and (2.10) hold, they remain true with $k^{\prime}<k$ in place of $k$, because the right-hand sides are increasing functions of $k$. Thus between $T$ consecutive values we find one for which $g_{2}(h)<k^{\prime} \sqrt{2 \delta}$, which implies

$$
g(h) \leq 2 m+g_{2}(h)<2 m+k \sqrt{2 \delta} .
$$

Next we show that with a suitable choice of the parameters almost all $k$-tuples are independent and quasiseparated.

2.6. Lemma. The number of $k$-tuples that are not $K$-independent is at most $(2 K+1)^{k} p^{k-1}$.

Proof. The number of possible equations (2.4) is at most $(2 K+1)^{k}$, since each coefficient lies between $-K$ and $K$, and an equation has at most $p^{k-1}$ solutions.

2.7. Lemma. The number of $k$-tuples that are not $K, L$-separated is at most

$$
(2 K+1)^{k}(2 L+1) p^{k-1} .
$$

Proof. The difference in comparison with the previous lemma is that we have to exclude equation (2.8), where there are $2 L+1$ possibilities for $y$, thus the total number of equations is bounded by $(2 K+1)^{k}(2 L+1)$.

2.8. LemmA. The number of $k$-tuples that are not $K, L, m$-quasiseparated is at most

$$
(2 K+1)^{k(m+1)}(2 L+1)^{m+1} p^{k-(m+1)} .
$$

Proof. Let $F(k, m, K, L)$ denote the number of $k$-tuples to be estimated. We know

$$
F(k, 0, K, L) \leq(2 K+1)^{k}(2 L+1) p^{k-1}
$$

from the previous lemma. Now we show

$$
F(k, m, K, L) \leq(2 K+1)^{k}(2 L+1) F(k-1, m-1, K, L) .
$$

These inequalities yield the lemma by an easy induction.

To prove (2.11), take a $k$-tuple that is not $K, L, m$-quasiseparated. It must satisfy an equation of type (2.8). The number of possible equations is $\leq(2 K+1)^{k}(2 L+1)$; we show that the number of such solutions of a fixed equation that are not quasiseparated is at most $F(k-1, m-1, K, L)$. Indeed, let $j$ be a subscript such that $x_{j} \neq 0$. Then $a_{j}$ is uniquely determined 
by $a_{1}, \ldots, a_{j-1}, a_{j+1}, \ldots, a_{k}$, which form a $(k-1)$-tuple that is not $K, L$, $m$ - 1-quasiseparated.

Proof of Theorem 1. Given $p$ and $\varepsilon$, we shall select a positive integer $k$, then a $k$-tuple of residues $a_{1}, \ldots, a_{k}$ and define $A$ by $(2.2)$. We use $k$ as a parameter which we shall optimize at the end; we assume $k \rightarrow \infty$ and $k=o(\log p)$.

We take four other parameters $K, L, m, K^{\prime}$ and try to find a $K^{\prime}$-independent $k$-tuple $a_{1}, \ldots, a_{k}$ such that $d a_{1}, \ldots, d a_{k}$ is $K, L, m$-quasiseparated for every $d \not \equiv 0(\bmod p)$. According to Lemmas 2.6 and 2.8 , such a $k$-tuple exists if

$$
\left(2 K^{\prime}+1\right)^{k} p^{k-1}+(p-1)(2 K+1)^{k m}(2 L+1)^{m} p^{k-m}<p^{k} .
$$

This is satisfied if

$$
\left(2 K^{\prime}+1\right)^{k}<p / 2
$$

and

$$
(2 K+1)^{k}(2 L+1)<p^{1-1 / m} / 2 .
$$

(2.12) is satisfied with $K^{\prime}=\left[p^{1 / k} / 3\right]$; we shall only need that $K^{\prime} \rightarrow \infty$, which follows from the assumption $k=o(\log p)$.

We define $A$ and $H$ by (2.2) and (2.3), with $Q=\varepsilon \sqrt{k}$. We use Theorem 2 to estimate the cardinality of $A(2.6)$ yields

$$
\frac{1}{p}|A|>1-\Phi(\sqrt{2} \varepsilon)-O\left(1 / k+1 / \sqrt{K^{\prime}}\right)>1 / 2-\varepsilon
$$

for large $p$, since both $k$ and $K^{\prime}$ tend to infinity.

$H$ is defined by the inequality $g(h)<2 Q$. We apply Statement 2.5. Since the conclusion we need is $g(h)<2 Q$, we put

$$
m=[Q / 2]=\left[\frac{\varepsilon}{2} \sqrt{k}\right]
$$

and $\delta=\varepsilon^{2} /(2 k)$. To satisfy (2.9), we define

$$
K=\left[(k \log k)^{2}\right] .
$$

With these parameters, Statement 2.5 is applicable not only to $g$ but to any of the functions $g_{d}(h)=g(h d)$, and we conclude that there is an element of $H$ among any $T$ consecutive terms of an arithmetic progression, where $T$ is given by (2.10). Our task is to minimize the quantity

$$
\frac{p}{L}\left(\frac{4 k}{\varepsilon^{2}}\right)^{2 k} \text {. }
$$

To satisfy $(2.13)$ we put

$$
L=\left[p^{1-1 / m} K^{-k} 3^{-k-1}\right]
$$


and then (2.15) becomes

$$
\leq 3^{k+1} p^{2 /(\varepsilon \sqrt{k})}\left(\frac{4 k^{2} \log k}{\varepsilon^{2}}\right)^{2 k} .
$$

The choice $k=\left[(\log p / \log \log p)^{2 / 3}\right]$ yields

$$
T<\exp c_{\varepsilon}(\log p \log \log p)^{2 / 3} .
$$

3. Large values of $f$. This section is devoted to the proof of Theorem 3 . Let $a_{1}, \ldots, a_{k} \in \mathbf{Z}_{p}, c_{1}, \ldots, c_{k}$ real numbers, $F(x)=\sum c_{j} e\left(a_{j} x / p\right), f(x)=$ $\operatorname{Re} F(x)=\sum c_{j} \cos \left(2 \pi a_{j} x / p\right), \sum\left|c_{j}\right|=S$.

We shall compare $f$ to a sum of independent random variables. Let $X_{1}, \ldots, X_{k}$ be independent random variables uniformly distributed on the circle $|z|=1, \xi_{j}=\operatorname{Re} X_{j}, Z=\sum c_{j} X_{j}, \zeta=\operatorname{Re} Z=\sum c_{j} \xi_{j}$.

We shall calculate moments of $f$ and $\zeta$. Write

$$
R_{u v}=\mathbf{E}\left(Z^{u} \bar{Z}^{v}\right), \quad r_{l}=\mathbf{E} \zeta^{l}=2^{-l} \sum_{v=0}^{l}\left(\begin{array}{l}
l \\
v
\end{array}\right) R_{v, l-v} .
$$

We are interested in the distribution of $f$ on $T$ consecutive numbers, say $y+1, \ldots, y+T$. Write

$$
\begin{aligned}
M_{u v} & =\frac{1}{T} \sum_{z=y+1}^{y+T} F(x)^{u} \overline{F(x)^{v}}, \\
m_{l} & =\frac{1}{T} \sum_{z=y+1}^{y+T} f(x)^{l}=2^{-l} \sum_{v=0}^{l}\left(\begin{array}{l}
l \\
v
\end{array}\right) M_{v, l-v} .
\end{aligned}
$$

3.1. Lemma. If the sequence $a_{1}, \ldots, a_{k}$ is $K, L$-separated, then for $u+v \leq$ $K$ we have

$$
\left|M_{u v}-R_{u v}\right| \leq \frac{p}{T L} S^{u+v} .
$$

Proof. Write

$$
\phi(b)=\frac{1}{T} \sum_{y=x+1}^{y+T} e(b x / p) .
$$

It is well known that

$$
\phi(b) \begin{cases}=1 & \text { if } b \equiv 0(\bmod p), \\ =0 & \text { if } b \neq \equiv 0, T=p, \\ \leq 1 / T\|b / p\| & \text { anyway, }\end{cases}
$$

where $\|\ldots\|$ means the distance from the nearest integer. We have

$$
M_{u v}=\sum c_{i_{1}} \ldots c_{i_{u}} \bar{c}_{j_{1}} \ldots \bar{c}_{j_{v}} \phi\left(a_{i_{1}}+\ldots+a_{i_{u}}-a_{j_{1}}-\ldots-a_{j_{v}}\right)
$$


and

$$
R_{u v}=\sum^{\prime} c_{i_{1}} \ldots c_{i_{u}} \bar{c}_{j_{1}} \ldots \bar{c}_{j_{v}},
$$

where the ' means that the summation is over those sequences of subscripts for which $\left(j_{1}, \ldots, j_{v}\right)$ is a permutation of $\left(i_{1}, \ldots, i_{u}\right)$ (thus it is empty unless $u=v)$. The assumption of $K, L$-separation means that the number $b=$ $a_{i_{1}}+\ldots+a_{i_{u}}-a_{j_{1}}-\ldots-a_{j_{v}}$ satisfies $\|b / p\| \geq L / p$ unless $\left(j_{1}, \ldots, j_{v}\right)$ is a permutation of $\left(i_{1}, \ldots, i_{u}\right)$. Consequently we have

$$
\left|M_{u v}-R_{u v}\right| \leq \frac{p}{T L} \sum\left|c_{i_{1}} \ldots c_{i_{u}} \bar{c}_{j_{1}} \ldots \bar{c}_{j_{v}}\right|=\frac{p}{T L} S^{u+v} .
$$

3.2. LemmA. If the sequence $a_{1}, \ldots, a_{k}$ is $K, L$-separated, then for $l \leq K$ we have

$$
\left|m_{l}-r_{l}\right| \leq \frac{p}{T L} S^{l} .
$$

Proof. This follows from the previous lemma, (3.1) and (3.2).

Proof of Theorem 3 . Assume indirectly that $f(x) \leq S(1-\delta)$ for $x=y+1, \ldots, y+T$. (The case of big negative values follows by considering the function $-f(x)$ similarly.) Then for every number

$$
U \geq \delta S / 2
$$

we have

$$
|f(x)+U| \leq U+S(1-\delta)
$$

for the same values of $x$. Consequently,

$$
\frac{1}{T} \sum(f(x)+U)^{l} \leq(U+S(1-\delta))^{l}
$$

for any even integer $l$. The sum on the left side of (3.8) is equal to

$$
\sum_{j=0}^{l} m_{j} U^{l-j}\left(\begin{array}{l}
l \\
j
\end{array}\right)=\sum_{j=0}^{l} r_{j} U^{l-j}\left(\begin{array}{l}
l \\
j
\end{array}\right)+\text { error }=\mathbf{E}\left((\zeta+U)^{l}\right)+\text { error. }
$$

By the previous lemma,

$$
\mid \text { error } \mid \leq \frac{p}{T L} \sum_{j=0}^{l} S^{j} U^{l-j}\left(\begin{array}{l}
l \\
j
\end{array}\right)=\frac{p}{T L}(S+U)^{l} \quad \text { if } l \leq K .
$$

We estimate the main term as follows:

$$
\mathbf{E}\left((\zeta+U)^{l}\right) \geq(U+S(1-\eta))^{l} \mathbf{P}(\zeta \geq S(1-\eta))
$$

with any $0<\eta<1$. Now $\zeta \geq S(1-\eta)$ certainly holds if $\xi_{j} \operatorname{sg} c_{j} \geq 1-\eta$ for all $j=1, \ldots, k$. The probability of one such event is

$$
\frac{1}{\pi} \arccos (1-\eta) \geq \frac{\sqrt{2}}{\pi} \sqrt{\eta} \geq \eta
$$


if $\eta<1 / 5$. This yields $\mathbf{P}(\zeta \geq S(1-\eta)) \geq \eta^{k}$, hence

$$
\mathbf{E}\left((\zeta+U)^{l}\right) \geq \eta^{k}(U+S(1-\eta))^{l} .
$$

Combining (3.7)-(3.10) we get the inequality

$$
(U+S(1-\delta))^{l} \geq \eta^{k}(U+S(1-\eta))^{l}-\frac{p}{T L}(S+U)^{l} .
$$

After introducing the parameter $\varrho=S /(U+S)$ and rearranging, this takes on the simpler form

$$
p /(T L) \geq \eta^{k}(1-\eta \varrho)^{l}-(1-\delta \varrho)^{l} .
$$

Condition (3.7) can be rewritten as

$$
\varrho \leq 2 /(2+\delta) .
$$

We put $\eta=\delta / 2$ into (3.12); the assumption $\delta<1 / 3$ guarantees $\eta<1 / 5$. We use the inequality $(1-t)^{2} \geq 1-2 t$ to obtain

$$
p /(T L) \geq \eta^{k} z-z^{2}
$$

with $z=(1-\eta \varrho)^{l}$. The quadratic function in (3.14) assumes its maximum at $z=\eta^{k} / 2$ and this choice yields

$$
p /(T L) \geq \eta^{2 k} / 2=(\delta / 2)^{2 k} / 2,
$$

which contradicts (2.10). The choice of $z$ determines $\varrho$, and it is compatible with (3.13) if and only if

$$
\eta^{k} / 2=\delta^{k} 2^{-k-1} \geq\left(1-\frac{\delta \varrho}{2}\right)^{l}=\left(\frac{2}{2+\delta}\right)^{l},
$$

or, equivalently,

$$
l \geq \frac{k \log (2 / \delta)+\log 2}{\log (1+\delta / 2)} .
$$

We have to find an even integer $l$ greater than the bound above but less than $K$; this is possible if $K$ is greater than the right side of $(3.15)+2$, which follows from (2.9).

3.3. Remark. Some of our calculations were far from optimal. Performing them with more precision would not, however, yield an essential improvement in the results. I do not know whether a more sophisticated method than this moment inequality could lead to sharper results and an improvement of the exponent in Theorem 1. I feel that most of the loss comes from the square-mean inequality used in (2.7).

4. The normal distribution of $f$. We prove Theorem 2. We retain the notations introduced at the beginning of the previous section. We shall compare the distribution of $f$ to that of $\zeta$, and $\zeta$ to the normal distribution. Since we are now interested in distribution on all residues, we put $T=p$. 
We also assume that our function is normalized so that $\sum c_{j}^{2}=2$, that is, $\sigma=1$. We recall the notation $\Delta=\max \left|c_{j}\right|$.

We use Esseen's famous inequality [3] in its simplest form:

4.1. Lemma. Let $G_{1}(x)$ and $G_{2}(x)$ be distribution functions with the corresponding characteristic functions $\gamma_{1}(t)$ and $\gamma_{2}(t)$. Assume that $G_{1}^{\prime}(x)$ exists and $G_{1}^{\prime}(x) \leq V$ for all $x$. Then

$$
\sup _{x}\left|G_{1}(x)-G_{2}(x)\right| \ll \frac{V}{T}+\int_{0}^{T} \frac{\left|\gamma_{1}(t)-\gamma_{2}(t)\right|}{t} d t
$$

where the implied constant is absolute.

First we consider $\zeta$. Let $\psi(t)=\mathbf{E} e^{i t \zeta}$ be its characteristic function, $P(x)=\mathbf{P}(\zeta \leq x)$ its distribution. that

$$
|\psi(t)| \leq \begin{cases}\exp \left(-\beta t^{2}\right) & \text { for }|t| \leq T_{0} / \Delta \\ (B \Delta|t|)^{-1 / \Delta^{2}} & \text { for }|t|>T_{0} / \Delta\end{cases}
$$

Proof. By the definition of $\zeta$ we have

$$
\psi(t)=\prod J\left(c_{j} t\right)
$$

where

$$
J(t)=\mathbf{E} e^{i t \xi_{j}}=\frac{1}{2 \pi} \int_{0}^{2 \pi} e^{i t \cos \alpha} d \alpha
$$

is a Bessel function. We only need the following properties of $J(t)$ :

$$
J(t)=1-t^{2} / 4+O\left(t^{4}\right)
$$

for small $t, J(t) \ll|t|^{-1 / 2}$ for large $t$, and $|J(t)|<1$ for all $t \neq 0$. Hence the function

$$
\beta_{T}=\min _{|t| \leq T} \frac{-\log |\psi(t)|}{t^{2}}
$$

satisfies

$$
\beta_{T} \geq \begin{cases}\frac{\log B T}{2 T^{2}} & \text { for } T>T_{0}, \\ \beta & \text { for } T \leq T_{0}\end{cases}
$$

with suitable constants $\beta>0, B>1$ and $T_{0}>1$. Observe that $|J(t)| \leq$ $\exp \left(-\beta_{T} t^{2}\right)$ for $|t| \leq T$ by the definition of $\beta_{T}$. Since $\left|c_{j} t\right| \leq \Delta|t|$ for all $j$, an application of this inequality for the numbers $c_{j} t$ with $T=\Delta|t|$ and a substitution to (4.3) yields

$$
|\psi(t)| \leq \exp \left(-\beta_{T} \sum\left(c_{j} t\right)^{2}\right)=\exp \left(-2 \beta_{T} t^{2}\right)
$$


(4.2) follows from this inequality and (4.6).

\subsection{Statement. We have}

$$
\max |P(x)-\Phi(x)| \ll \Delta^{2} .
$$

Pr o of. By Lemma 4.1, the left side is

$$
\ll \int_{0}^{\infty} \frac{\left|\psi(t)-e^{-t^{2} / 2}\right|}{t} d t .
$$

Let $T_{1}>0$ be a number such that (4.4) holds for $|t|<T_{1}$. Applying (4.4) to each factor we obtain

$$
\psi(t)=e^{-t^{2} / 2}+O\left(t^{4} \sum c_{j}^{4}\right)=e^{-t^{2} / 2}+O\left(\Delta^{2} t^{4}\right),
$$

for $|t| \leq T_{1} / \Delta$, since

$$
\sum c_{j}^{4} \leq\left(\max c_{j}\right)^{2} \sum c_{j}^{2} \leq 2 \Delta^{2} .
$$

For $|t| \leq T_{1} / \Delta$ this implies

$$
\psi(t)-e^{-t^{2} / 2} \ll \Delta^{2} t^{2} e^{-t^{2} / 2},
$$

which implies that the contribution of $|t| \leq T_{1} / \Delta$ to $(4.8)$ is $O\left(\Delta^{2}\right)$. For $|t|>T_{1} / \Delta$ we apply Lemma 4.2 to $\psi$ and obtain the same bound after a routine calculation.

4.4. Remark. The bound $O\left(\Delta^{2}\right)$ is sharp. We could immediately deduce the weaker bound $O(\Delta)$ from the Berry-Esseen inequality $[1,3]$. The improvement is due mainly to the fact that not only the first but also the third moments of $\xi_{j}$ vanish.

Now we turn to comparing $\zeta$ and $f$.

4.5. Lemma. If the sequence $a_{1}, \ldots, a_{k}$ is $K$-independent, then $m_{l}=r_{l}$ for $l \leq K$.

The proof is analogous to that of Lemma 3.2, we just apply the second case of (3.4) instead of the third.

Recall that $S=\sum\left|c_{j}\right|$.

4.6. Lemma. Let $l=2 u$ be an even positive integer. Then

$$
r_{l} \leq \min \left(S^{l}, u !\right) .
$$

Proof. We always have $|\zeta| \leq S$, thus $r_{l} \leq S^{l}$ is obvious. To prove $r_{l} \leq u$ ! recall that by $(3.1)$ and $(3.5)$

$$
r_{l}=2^{-l} \sum c_{i_{1}} \ldots c_{i_{u}} \bar{c}_{j_{1}} \ldots \bar{c}_{j_{u}}
$$


where the summation is over those sequences of subscripts for which $\left(j_{1}, \ldots, j_{u}\right)$ is a permutation of $\left(i_{1}, \ldots, i_{u}\right)$. Since a fixed sequence $\left(i_{1}, \ldots, i_{u}\right)$ has at most $u$ ! permutations, we obtain

$$
r_{l} \leq 2^{-l} u ! \sum\left|c_{i_{1}} \ldots c_{i_{u}}\right|^{2}=2^{-l} u !\left(\sum\left|c_{j}\right|^{2}\right)^{u}=u !
$$

4.7. Lemma. If $\Delta<1 / 2$, then $P^{\prime}(x)$ is bounded by an absolute constant.

Proof. This follows from the familiar inequality

$$
P^{\prime}(x) \leq \int_{-\infty}^{\infty}|\psi(t)| d t
$$

and Lemma 4.2.

4.8. Statement. If $\Delta<1 / 2$, then

$$
\frac{1}{p} \sum_{f(x) \leq t \sigma} 1-P(t) \ll \min \left(\frac{1}{\sqrt{K}}, \frac{S}{K}\right) .
$$

Pro of. Denote this difference by $R$. By the previous lemma and Lemma 4.1 we have

$$
R \ll \frac{1}{T}+\int_{0}^{T} \frac{|\psi(t)-\chi(t)|}{t} d t
$$

where

$$
\chi(t)=\frac{1}{p} \sum_{x=1}^{p} e^{i t f(x)}
$$

For every real $t$ and positive integer $l$ we have

$$
e^{i t}=\sum_{j=1}^{l-1} \frac{(i t)^{j}}{j !}+\vartheta \frac{t^{l}}{l !}
$$

with $|\vartheta| \leq 1$. Applying this formula both to $e^{i t \zeta}$ and $e^{i t f(x)}$ we obtain

$$
\psi(t)=\sum_{j=1}^{l-1} \frac{r_{j}}{j !}(i t)^{j}+\vartheta r_{l} \frac{|t|^{l}}{l !}, \quad \chi(t)=\sum_{j=1}^{l-1} \frac{m_{j}}{j !}(i t)^{j}+\vartheta m_{l} \frac{|t|^{l}}{l !} .
$$

In view of Lemma 4.5 we have for even $l \leq K$

$$
|\psi(t)-\chi(t)| \leq 2 r_{l} \frac{t^{l}}{l !} .
$$

Substituting this into (4.10) we find

$$
R \ll \frac{1}{T}+\frac{r_{l}}{l !} \frac{T^{l}}{l} .
$$


The optimal choice is $T=\left(l ! / r_{l}\right)^{1 /(l+1)}$ and it yields

$$
R \ll\left(\frac{r_{l}}{l !}\right)^{1 /(l+1)} \ll \frac{r_{l}^{1 / l}}{l} \min \left(\frac{S}{l}, \frac{1}{\sqrt{l}}\right)
$$

by Lemma 4.6. The statement follows by taking the maximal admissible value $l=2[K / 2]$.

Proof of Theorem 2. For $\Delta<1 / 2$ the conclusion follows from Statements 4.3 and 4.8 , and for $\Delta \geq 1 / 2$ it holds obviously.

5. Concluding remarks. In a typical problem of combinatorial number theory, the extremal sets are either very regular, or random sets. Our case is different. If we take a random subset of $\mathbf{Z}_{p}$, then with probability 1 we have $A+A=\mathbf{Z}_{p}$. If $A$ is an arithmetic progression of $k$ elements, then $A+A$ is also an arithmetic progression itself. "Multidimensional" arithmetic progressions are somewhat better. Say, put

$$
A=\left\{n: n=x_{1} d_{1}+\ldots+x_{k} d_{k}, 0 \leq x_{i} \leq m-1\right\},
$$

a set of $m^{k}$ elements if all of them are different. Here $A+A$ contains arithmetic progressions of $2 m-1$ elements but no longer if, say, $d_{j+1} / d_{j}>$ $2 m$. This gives $n^{\delta}$ for the length if $|A|=c N, A \subset[1, N]$, where $\delta=\delta(c) \rightarrow 0$ as $c \rightarrow 0$, still far from (1.1).

Another application of a niveau set of a trigonometric polynomial to an additive problem was given in [4].

\section{References}

[1] A. C. Berry, The accuracy of the Gaussian approximation to the sum of independent variables, Trans. Amer. Math. Soc. 49 (1941), 122-136.

[2] J. Bourgain, On arithmetic progressions in sums of sets of integers, in: A Tribute to Paul Erdős (A. Baker, B. Bollobás, A. Hajnal, eds.), Cambridge Univ. Press, Cambridge 1990, 105-109.

[3] C. G. Esseen, Fourier analysis of distribution functions. A mathematical study of the Laplace-Gaussian law, Acta Math. 77 (1945), 1-125.

[4] I. Z. Ruzsa, Essential components, Proc. London Math. Soc. 54 (1987), 38-56.

MATHEMATICAL INSTITUTE

HUNGARIAN ACADEMY OF SCIENCES

BUDAPEST, PF. 127, H-1364 HUNGARY 